\title{
Nanoparticle formation in a cavitation bubble after pulsed laser ablation in liquid studied with high time resolution small angle $x$-ray scattering
}

\author{
Shyjumon Ibrahimkutty, ${ }^{1}$ Philipp Wagener, ${ }^{2}$ Andreas Menzel, ${ }^{3}$ Anton Plech, ${ }^{1, a)}$ \\ and Stephan Barcikowski ${ }^{2, b)}$ \\ ${ }^{1}$ Institute for Synchrotron Radiation, Karlsruhe Institute of Technology, Postfach 3640, \\ D-76021 Karlsruhe, EU, Germany \\ ${ }^{2}$ Technical Chemistry I and Center for Nanointegration Duisburg-Essen (CeNIDE), \\ University of Duisburg-Essen, Universitätsstrasse 7, D-45141 Essen, EU, Germany \\ ${ }^{3}$ Paul Scherrer Institut, CH-5232 Villigen PSI, Switzerland
}

(Received 25 April 2012; accepted 21 August 2012; published online 4 September 2012)

\begin{abstract}
We investigated nanoparticle formation after pulsed laser ablation in liquid using time-resolved small angle x-ray scattering. Laser ablation of a gold target in water induces a cavitation bubble in which two different particle species could be identified at maximum bubble extension: (i) primary particles of about $8-10 \mathrm{~nm}$ diameter, which show a smooth concentration gradient starting from the target and can also be found outside the cavitation bubble in the free liquid and (ii) secondary particles in the range of $45 \mathrm{~nm}$ diameter which have highest concentration in the upper part of the cavitation bubble but do not penetrate into the liquid. (c) 2012 American Institute of Physics. [http://dx.doi.org/10.1063/1.4750250]
\end{abstract}

Laser ablation in liquid represents an alternative route to produce nanoparticles useful in a large variety of applications $^{1}$ and is a current and fast growing field of research. ${ }^{2}$ Compared to wet chemical synthesis, pure electrostatically stabilized nanoparticles free of any ligands or impurities of reactions products and educts are accessible. These particles are very interesting to be conjugated with biomolecules ${ }^{3}$ or polymers ${ }^{4}$ reaching a high conjugation efficiency and surface coverage. However, the detailed formation process of nanoparticles after laser ablation is still unclear. Besides experimental findings that nanoparticles are formed by liquid-droplet ejection, ${ }^{5}$ it is generally agreed that the laserinduced cavitation bubble plays an important role during nanoparticle formation. The confinement inside the bubble provides trapping of the ablated material, nucleation, and final solidification of nanoparticles. ${ }^{6-9}$ Unfortunately, the direct physical insight of former investigations is limited because those are usually based on indirect methods like post-mortem analysis of fabricated nanoparticles or measurement of laser-induced cavitation bubble dynamics using shadowgraphy ${ }^{6,10}$ or light scattering techniques. Due to the optically dense interface between cavitation bubble and liquid, a direct observation of particle formation dynamics inside the bubble is not possible by means of optical spectroscopy. Furthermore, scattering of laser light is less sensitive to small nanoparticles and clusters. A more promising approach is based on the use of $\mathrm{x}$-rays, which can penetrate the cavitation bubble and identify the participating species by scattering even in case of small particles.

Recently, Lavisse et al. showed that in-situ small-angle $\mathrm{x}$-ray scattering (SAXS) is a powerful method to characterize the particle formed after pulsed laser ablation in ambient air. ${ }^{11}$ However, detailed analysis of formation mechanisms are limited by lack of time and sufficient spatial resolution.

\footnotetext{
${ }^{a)}$ Electronic mail: anton.plech@kit.edu.

b)Electronic mail: stephan.barcikowski@uni-due.de.
}

Within this communication, we present an approach based on SAXS which allows direct observation of the particle formation process inside the cavitation bubble. With this in-situ characterization, we aim to address the current discussion of nanoparticle formation mechanisms after pulsed laser ablation in liquid.

The experiment was performed at the cSAXS beamline at the Swiss Light Source (13.6 keV photons). The used experimental scheme is shown in Fig. 1. We used a Nd:YAG laser $(1064 \mathrm{~nm}, 6 \mathrm{~ns}, 10 \mathrm{~mJ}, 200 \mathrm{~Hz}$, EdgeWave HD-40I-E) to ablate a gold ribbon in a water-filled ablation chamber. The laser was focused by a spherical $75 \mathrm{~mm}$ lens, resulting in a spot size of about $100 \mu \mathrm{m}$. The gold ribbon was continuously transported perpendicular to the laser beam axis by precise rolls and a Teflon support to provide a fresh target for every laser shot. At the same time, a concurrent flow of deionized water $(25 \mathrm{l} / \mathrm{h})$ was provided in the same direction as the ribbon movement in order to exchange the liquid above the ablated spot between the shots. This flow speed ensured the replacement of about $1 \mathrm{~mm}$ of liquid along the flow direction through a cross section of 3 (distance between Kapton windows) by $10 \mathrm{~mm}$ (water height) within the $5 \mathrm{~ms}$ between two laser shots. At the same time, a possible shear deformation of the cavitation bubbles within the $230 \mu$ s lifetime is much less than the bubble extension. For the detection of ablated species with spatial specificity inside the bubble region, a horizontal $\mathrm{x}$-ray beam intersects the ablation region with an $\mathrm{x}$-ray focus size of $6 \mu \mathrm{m}$ in the vertical direction and $23 \mu \mathrm{m}$ horizontally.

The scattered intensity was recorded by a pixel detector (Pilatus $2 \mathrm{M}){ }^{12}$ The central beam block was equipped with a $\mathrm{p}$-i-n diode, which allowed recording the $\mathrm{x}$-ray transmission (about $40 \%$ in average) through the sample with high time resolution on an oscilloscope (LeCroy). The x-ray beam was centered on the laser path. The cavitation bubble was scanned by vertical movement of the set-up relative to the fixed x-ray beam (Fig. 1). 

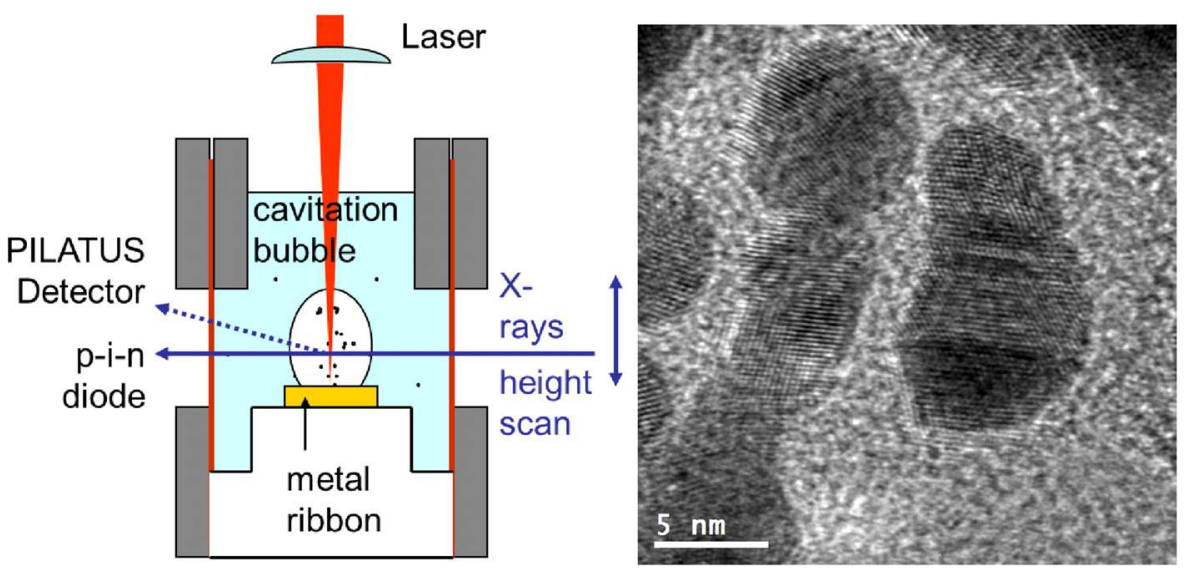

FIG. 1. Sketch of the setup with the metal ribbon inside the water chamber. Two $75 \mu \mathrm{m}$ Kapton foils seal the chamber and act as $\mathrm{x}$-ray windows. Right: TEM microscopy image of the final particles as collected from the flush water.
Typical $\mathrm{x}$-ray transmission changes through the water column are shown in Fig. 2. The curves are averages over all laser pulses used for the averaging of the scattering data at a given height as explained below. During bubble formation, the $\mathrm{x}$-ray transmission is increased, which is directly indicative of a low-density bubble volume formed. Thus, we can deduce both the bubble kinetics and the maximum bubble extension from the oscilloscope traces. The bubble apex is seen at $1.15 \mathrm{~mm}$ above the target (Fig. 2, inset, zero crossing of the transmission curve). The absolute bubble thickness in the direction of the x-ray beam cannot be evaluated quantitatively due to bulging of the Kapton window. This effect can also be seen above the bubble, where at a height of 1.1 to $1.4 \mathrm{~mm}$, the transmission through the column is transiently reduced, as the bubble below drives a pressure front. The shoulder at $230 \mu$ s shows the appearance of a second bubble, which is a rebound after the first collapse, typical for laserinduced cavitation bubbles. ${ }^{13,14}$ Its maximum height can be deduced from the cut in the inset, showing an extension of $0.7-0.8 \mathrm{~mm}$ above the target.

The x-ray small-angle scattering was recorded by timegated detection. The detector registers were only activated during a given electronic gate, which was synchronized with the laser pulses. Reading out of the pixel counters was done typically after accumulating $2 \times 10^{4}$ shots. The gate was

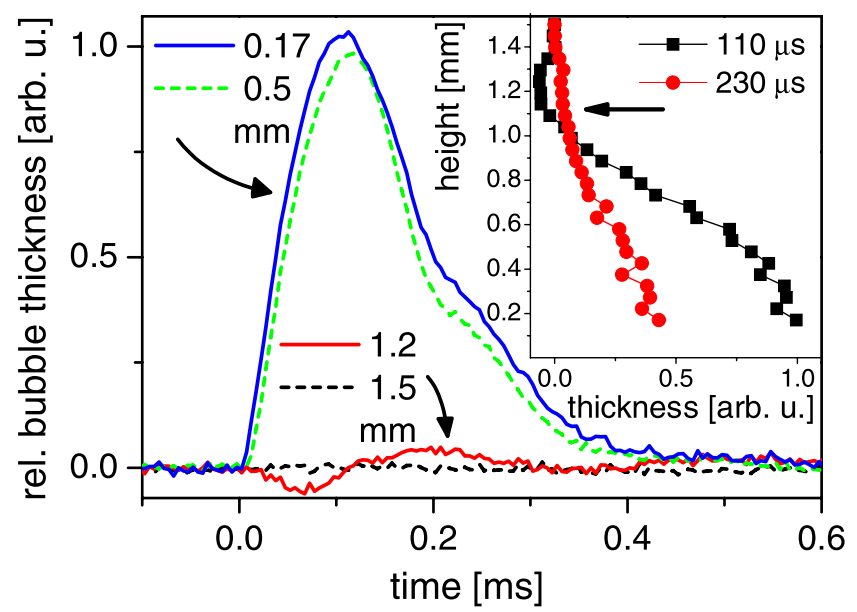

FIG. 2. X-ray transmission change through the laser-excited bubble as function of time and height above the target. The inset displays the relative bubble thickness as function of height for two time delays after laser excitation. chosen to be $80 \mu$ s which is shorter than the bubble lifetime. Scanning of either the position above the target or the time delay allowed the particle size distribution to be mapped as function of time and position within the ablation plume.

SAXS yields signals, which are Fourier transforms of the density-density correlations in the sample. Symmetric particles of defined size give rise to detailed scattering patterns, while in the general case of broad particle size and shape distributions, the scattering curves show only a few features in the scattering distribution. Among them is the characteristic intensity decay as function of the scattering vector $Q=$ $4 \pi / \lambda \cdot \sin (2 \theta / 2)(\lambda$ being the $x$-ray wavelength and $2 \theta$ the scattering angle) which is generally expressed by the Guinier law. A corresponding unified description for the scattering intensity $I(Q)$ has been developed for particle size distributions, in particular with several hierarchy levels $i$ as ${ }^{15,16}$

$$
I(Q)=\sum_{i} N_{i} \Delta \rho_{i} V_{i}^{2} \exp \left(-Q^{2} R_{g, i}^{2} / 3\right)+\sum_{i} B\left(Q_{i}^{\star}\right)^{-d_{f, i}},
$$

with $N_{i}$ as number densities, $\Delta \rho_{i}$ is the excess electron density relative to water, $V_{i}$ is the particle volume, $R_{g, i}$ is radius of gyration, $Q_{i}^{\star}=Q / \operatorname{erf}\left(Q R_{g} / \sqrt{6}\right)^{3}$ is the lower limit of a power law decay region, and $d_{f, i}$ is the mass fractal dimension. For massive particles with sharp interface, the second term with the prefactor B reduces to $2 \pi N_{i} \Delta \rho_{i}^{2} Q^{-4}$. The important feature for size determination is that each hierarchy level is reflected by a intensity maximum at a given $\mathrm{Q}$ value, which is inversely proportional to the particle size.

Scattering distributions at selected heights above the target are displayed in Fig. 3, which are multiplied by $Q^{2}$ (Kratky representation). The curves are taken for a time delay at the largest bubble extension (at $110 \mu \mathrm{s}$ ). The scattering signal without ablation pulse was subtracted, as the liquid in front and behind the bubble may also contain nanoparticles and contribute to the scattering. In practice, this was done by taking a scattering distribution right before the arrival of the laser pulse at the target $(-30 \mu \mathrm{s})$. It was not attempted to deduce a width of the size distribution from parameters in Eq. (1), as the calculation of the polydispersity index ${ }^{16}$ would require to determine each $\mathrm{B}\left(\mathrm{Q}_{i}\right)$ independently. The appearance of two distinct peaks shows two clearly distinguishable size populations.

By this method, we were able to identify two different species within the ablation region: small primary particles of 


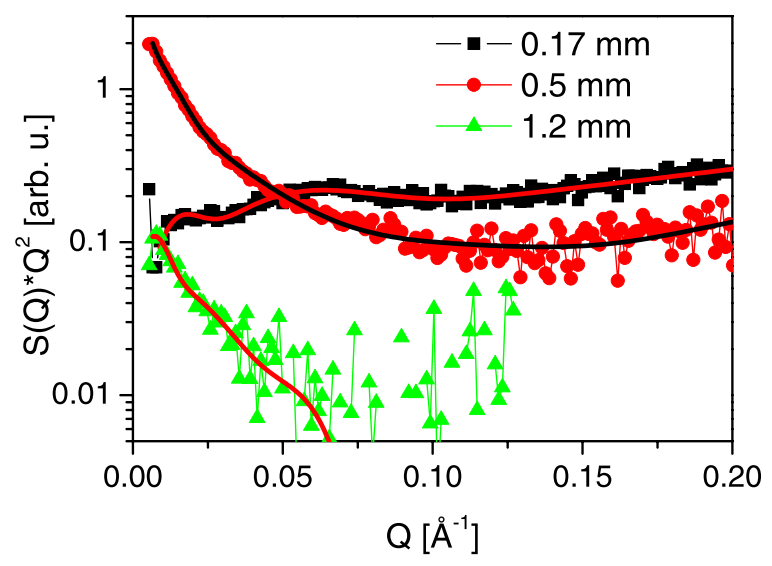

FIG. 3. Right: Selection of scattering curves at different heights above the target in the Kratky representation together with fits to the data according to Eq. (1).

about $8-10 \mathrm{~nm}$ and secondary particles of about $45 \mathrm{~nm}$ average diameter. Scanning over the total height of the bubble allows mapping of the mass distribution within the cavitation bubble. Figure 4 shows the mass distribution of primary and secondary particles (agglomerates) as a function of height above the target. Instead of using the fit values for the number densities in Eq. (1), it is more convenient to integrate $I(Q) * Q^{2}$ over the appropriate $\mathrm{Q}$ range proportional to $R_{g}^{-1}$ to derive a shape-invariant quantity that is related to the integrated particle mass within the illuminated volume. ${ }^{17}$

As can be seen in Fig. 4, the primary particle abundance decays smoothly towards the top of the bubble. As the bubble thickness reduces towards the top, this nevertheless means that particles are accumulated in the upper part. Furthermore, the primary particles may cross the bubble interface as a significant amount of primary particles are detected outside bubble within the free liquid. Indeed initial plasma velocities in vacuum phase ablation within the expansion phase are found to be $10^{8} \mathrm{~mm} / \mathrm{s}^{10}$ This transport would allow to inject atomic species into the liquid, which subsequently cluster on a microsecond time scale. ${ }^{18}$

The average primary particle size is almost constant along the cavitation bubble. There is just a slight decrease

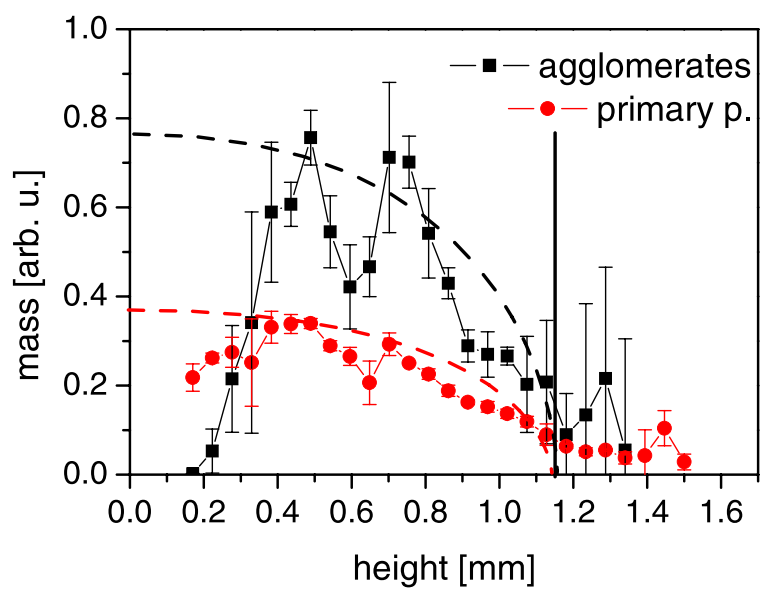

FIG. 4. Integrated intensity (invariant) as a measure for particle mass within the Guinier region of the primary particles (circles) and agglomerates (squares). The vertical line marks the position of the maximum bubble extension. Dashed lines indicate the relative bubble thickness. The error bars indicate the significance of the invariant over the background before laser impact $(-30 \mu \mathrm{s})$ in terms of counting statistics. from 10 to $8 \mathrm{~nm}$ average diameter with height. This decrease may be explained by a higher density and coalescence probability, possibly with atomic or cluster species, close to the target. In literature, rapid formation of primary particles is frequently discussed. For instance, results of MD simulations, ${ }^{19}$ kinetic approaches, ${ }^{20}$ or scattering experiments ${ }^{21}$ point towards this. The present results give experimental evidence that those often postulated small primary particle $(<10 \mathrm{~nm})$ are formed already at early stages within the laserinduced cavitation bubble.

It is noteworthy that the detected gold nanoparticles within the cavitation bubble are significantly larger than the smallest nanoparticles that are laser-fabricated in presence of a stabilizing agent under similar experimental conditions. ${ }^{20}$ Although the scattering weight factor (Eq. (1)) reduces the visibility of smaller particles, the main population of $8-10 \mathrm{~nm}$ particles is still evident. Size quenching through stabilizing agents may happen efficiently for those particles, which have crossed the vapor-liquid interface. A direct answer to this question may be obtained in future studies in the presence of surfactants. Additionally, most experiments described in literature are usually carried out in a stationary batch ablation chamber. This results in post-irradiation and subsequent size-reduction of laser-generated nanoparticles. ${ }^{20}$ In our set-up, the analyzed liquid is completely exchanged between two shots and no laser-induced size reduction decreases the primary particle size later. After release from the cavitation bubble, the primary nanoparticles are expected to undergo slow growth ${ }^{22}$ and agglomeration. TEM analysis of the flush water (see Fig. 1, right side) from the experiment reveals the existence of extended secondary particles built from small primary particles $(<10 \mathrm{~nm})$. The TEM images should be nevertheless regarded with precaution as the particles may have undergone further ripening.

We observe secondary particles of $45 \mathrm{~nm}$ average size already forming within the cavitation bubble by collisions of primary particles. The large secondary particles are not detected close to the target surface, but accumulate in the middle of the bubble. This is due to both the reaction probability with time and height and the confinement and cooling down of the small particles near the vapor-liquid interface. ${ }^{23}$ The majority of ablated mass is accumulated within the primary particles and just a few percent $(0.1 \%-10 \%)$ of the detected mass within the cavitation bubble is related to secondary particles. Furthermore, the secondary particles seem not to penetrate the bubble interface at this point of time as this particle mass fraction is not significantly detected outside the cavitation bubble. Soliman et al. ${ }^{7}$ detect large particles by light scattering before the bubble expansion and around collapse. This could be explained in our case by emitted agglomerates eventually disintegrating to primary particles at the time of maximum bubble expansion.

In conclusion, time-resolved SAXS during laser ablation of gold in water allows to study the particle formation process in-situ and inside the cavitation bubble with well-defined conditions, e.g., in the absence of secondary particle fragmentation in the laser beam. ${ }^{24}$ We have clarified that nanoparticle formation takes place within a hemi-spherical cavitation bubble. Our results are depicted in Fig. 5: at maximum bubble extension, two different species can be identified. Primary 

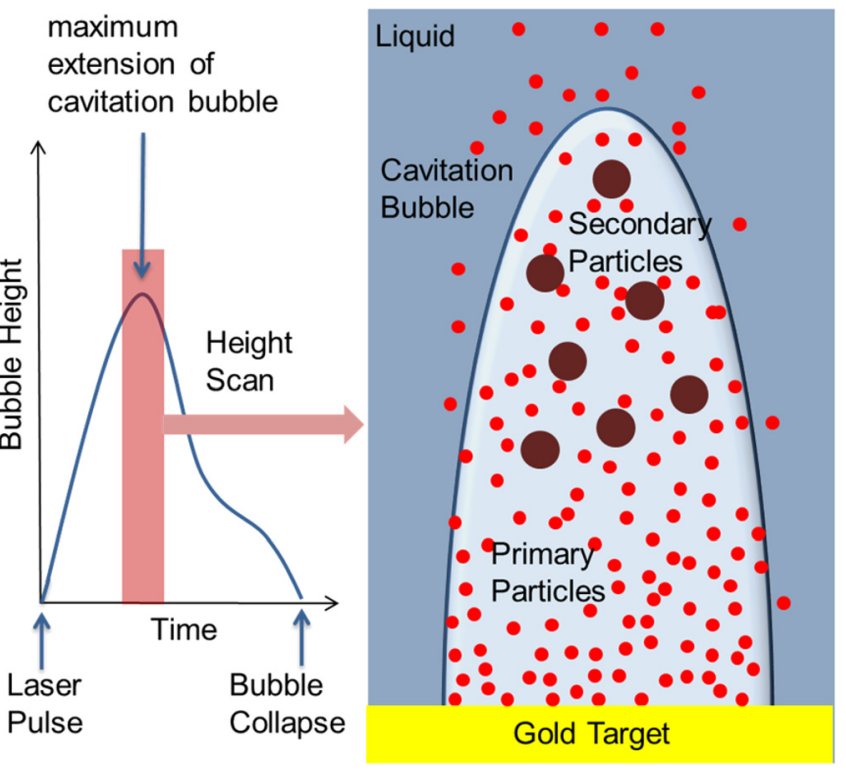

FIG. 5. Sketch of involved species in laser-induced cavitation bubble during its largest extension.

particles of about $8-10 \mathrm{~nm}$ average diameter distributed all over the bubble volume. Those particles may penetrate the bubble interface and can be detected even outside the cavitation bubble before its collapse. Collisions of primary particles result in secondary particles with $45 \mathrm{~nm}$ average diameter, which are trapped within the bubble and reach a maximum in particle mass at the upper part of the bubble.

This work is supported by the Center for Applied Photonics Baden-Württemberg and the German Research Foundation (DFG) within the priority program 1327 . Beamtime at the SLS and funding from the European Community's Seventh Framework Programme (FP7/2007-2013) under Grant Agreement No. 226716 is gratefully acknowledged, with special thanks to the excellent support by C. Kramer, M. Caspar, B. Henrich, and H. Billich. A.P. acknowledges the Heisenberg fellowship by DFG.
${ }^{1}$ H. Zeng, X. W. Du, S. C. Singh, S. A. Kulinich, S. Yang, J. He, and W. Cai, Adv. Funct. Mater. 22, 1333 (2012).

${ }^{2}$ S. Barcikowski, F. Devesa, and K. Moldenhauer, J. Nanopart. Res. 11, 1883 (2009)

${ }^{3}$ S. Petersen and S. Barcikowski, J. Phys. Chem. C 113, 19830 (2009).

${ }^{4}$ P. Wagener, S. Faramarzi, A. Schwenke, R. Rosenfeld, and S. Barcikowski, Appl. Surf. Sci. 257, 7231 (2011).

${ }^{5}$ E. Stratakis, M. Barberoglou, C. Fotakis, G. Viau, C. Garcia, and G. A. Shafeev, Opt. Express 17, 12650 (2009).

${ }^{6}$ K. Sasaki, T. Nakano, W. Soliman, and N. Takada, Appl. Phys. Express 2, 046501 (2009).

${ }^{7}$ W. Soliman, N. Takada, and K. Sasaki, Appl. Phys. Express 3, 035201 (2010).

${ }^{8}$ T. Tsuji, D.-H. Thang, Y. Okazaki, M. Nakanishi, Y. Tsuboi, and M. Tsuji, Appl. Surf. Sci. 254, 5224 (2008).

${ }^{9}$ P. Wagener, A. Schwenke, B. N. Chichkov, and S. Barcikowski, J. Phys. Chem. C 114, 7618 (2010).

${ }^{10}$ S. S. Mao, X. Mao, R. Greif, and R. E. Russo, Appl. Phys. Lett. 77, 2464 (2000).

${ }^{11}$ L. Lavisse, J.-L. Le Garrec, L. Hallo, J.-M. Jouvard, S. Charles, J. Perez, J. B. A. Mitchell, J. Decloux, M. Girault, V. Potin, H. Andrzejewski, M. C. M. de Lucas, and S. Bourgeois, Appl. Phys. Lett. 100, 164103 (2012).

${ }^{12}$ B. Henrich, A. Bergamaschi, C. Broennimann, R. Dinapoli, E. Eikenberry, I. Johnson, M. Kobas, P. Kraft, A. Mozzanica, and B. Schmitt, Nucl. Instrum. Methods A 607, 247 (2009).

${ }^{13}$ A. Vogel, N. Linz, S. Freidank, and G. Paltauf, Phys. Rev. Lett. 100, 038102 (2008).

${ }^{14}$ V. Kotaidis and A. Plech, Appl. Phys. Lett. 87, 213102 (2005).

${ }^{15}$ G. Beaucage, J. Appl. Crystallogr. 28, 717 (1995).

${ }^{16}$ M. Sztucki, T. Narayanan, and G. Beaucage, J. Appl. Phys. 101, 114304 (2007).

${ }^{17}$ A. Plech, A. Siems, V. Kotaidis, and M. Sztucki, Phys. Chem. Chem. Phys. 10, 3888 (2008).

${ }^{18}$ A. Plech, V. Kotaidis, M. Lorenc, and M. Wulff, Chem. Phys. Lett. 401, 565 (2005).

${ }^{19}$ T. E. Itina, J. Phys. Chem. C 115, 5044 (2011).

${ }^{20}$ F. Mafuné, J. Kohno, Y. Takeda, and T. Kondow, J. Phys. Chem B 105, 5114 (2001).

${ }^{21}$ W. Soliman, N. Takada, and K. Sasaki, Jpn. J. Appl. Phys., Part 1 50, 108003 (2011).

${ }^{22}$ C. Sajti, A. Barchanski, P. Wagener, S. Klein, and S. Barcikowski, J. Phys. Chem. C 115, 5094 (2011).

${ }^{23}$ S. Besner and M. Meunier, Laser Precision Manufacturing, edited by K. Sugioka, M. Meunier, and A. Piquet (Springer, Berlin-Heidelberg, 2010), pp. 163-187.

${ }^{24}$ S. Besner, A. V. Kabashin, and M. Meunier, Appl. Phys. Lett. 89, 233122 (2006). 